Kansas State University Libraries

New Prairie Press

\title{
COMPARING ESTIMATION PROCEDURES FOR DOSE-RESPONSE FUNCTIONS
}

William J. Price

Bahman Shafii

Ken B. Newman

Shane Early

Joseph P. McCaffrey

See next page for additional authors

Follow this and additional works at: https://newprairiepress.org/agstatconference

Part of the Agriculture Commons, and the Applied Statistics Commons

\section{(c) (1) $\Theta$}

This work is licensed under a Creative Commons Attribution-Noncommercial-No Derivative Works 4.0 License.

\section{Recommended Citation}

Price, William J.; Shafii, Bahman; Newman, Ken B.; Early, Shane; McCaffrey, Joseph P.; and Morra, Matthew, J. (2003). "COMPARING ESTIMATION PROCEDURES FOR DOSE-RESPONSE FUNCTIONS," Conference on Applied Statistics in Agriculture. https://doi.org/10.4148/2475-7772.1177

This is brought to you for free and open access by the Conferences at New Prairie Press. It has been accepted for inclusion in Conference on Applied Statistics in Agriculture by an authorized administrator of New Prairie Press. For more information, please contact cads@k-state.edu. 


\section{Author Information}

William J. Price; Bahman Shafii; Ken B. Newman; Shane Early; Joseph P. McCaffrey; and Matthew, J. Morra 


\title{
COMPARING ESTIMATION PROCEDURES FOR DOSE-RESPONSE FUNCTIONS
}

\author{
William J. Price, Bahman Shafii \\ Statistical Programs \\ University of Idaho, Moscow, ID 83844 \\ Ken B. Newman \\ Centre for Research into Ecological and Environmental Modelling \\ University of St Andrews, St Andrews, Fife KY169LZ, Scotland \\ Shane Early, Joseph P. McCaffrey, Matthew, J. Morra \\ Department of Plant, Soil, and Entomological Sciences \\ University of Idaho, Moscow, ID 83844
}

\begin{abstract}
The dose-response design is often used in agricultural research when it is necessary to measure a biological response at various levels of an experimental factor. This type of problem is common in chemical and pesticide research, however, it can also occur in other disciplines such as plant, animal, soil, and environmental sciences. While the analysis of dose-response data usually involves fitting a regression curve, the primary objective often centers on the estimation of doserelated percentiles such as the $\mathrm{LD}_{50}$ or $\mathrm{LC}_{50}$. These measures are useful for comparing the relative efficacy of various treatments, however, the estimation of the specified percentiles is not always straightforward. Traditional methodology has relied on inverted solutions or asymptotic theory for statistical inference. More recently, computer intensive methods have been used to model dose-response relationships and can be more appropriate than traditional methods in some situations. This paper examines both the traditional and modern approaches to estimating doseresponse functions as they apply to binomial data. The techniques will be demonstrated using mortality data collected on black vine weevil eggs exposed to an organic pesticide treatment.
\end{abstract}

Key words: Linearized Probit Analysis, Generalized Nonlinear Models, Bayesian Estimation

\section{INTRODUCTION}

Dose-response problems commonly occur in agricultural research. Examples can be found in plant science, soil science, entomology, and animal science (see for example Dungan, et al. 2001; Hernandez-Sevillano, et al., 2001; Turner, et al., 1995) . The general framework of doseresponse modeling encompasses many types of problems including the effects of time, e.g. germination and emergence responses, or environmental effects, such as temperature and chemical exposure. Dose-response modeling also arises in the related areas of bioassay, calibration, and standard curve estimation where the objective is to determine an unknown dose given an observed response.

Graphically, dose-response problems are often expressed as nonlinear curves. Over a range of dosages, the measured response, will often change in either an increasing or decreasing 
sigmoidal fashion, although the response may also follow other asymptotic patterns, depending on the research problem. The responses, in either case, may be considered to originate from a continuous distribution, e.g. normal, log-normal, gamma, etc., or possibly from a discrete distribution such as the binomial, multinomial or Poisson.

Estimation of the dose-response functions can be carried out using linear or nonlinear regression techniques. The resulting estimated curves are not only useful for describing the overall dose-response process, but the estimated parameter values may provide valuable information relevant to the problem under investigation. For example, the objective of doseresponse analysis typically involves the estimation of an unknown dose which corresponds to a predetermined level of response, say $50 \%$. These estimated percentiles are usually referred to as the lethal dose $50 \%\left(\mathrm{LD}_{50}\right)$, the lethal concentration $50 \%\left(\mathrm{LC}_{50}\right)$, the effective concentration $50 \%$ $\left(\mathrm{EC}_{50}\right)$, etc., dependent on the context of the problem and represent the dosage required to achieve a $50 \%$ response rate. While estimation of other percentiles may be desirable for the problem, the $50^{\text {th }}$ percentile is traditionally used as a measure of efficacy and often is the easiest to compute. Estimation of a dose conditional on a fixed response can be problematic, however, since it essentially involves working the regression problem backwards, i.e. an inverted solution usually having an unknown distribution which must be approximated. Thus, any statistical inferences or contrasts on these quantities will also be approximated.

This paper will outline and demonstrate several estimation methods for binomial doseresponse data. The methods considered will cover traditional approaches including least squares and maximum likelihood, as well as more contemporary solutions consisting of generalized nonlinear models and Bayesian estimation techniques.

\section{METHODS}

\section{Traditional Estimation Techniques}

\subsection{Linearized Least Squares Probit Analysis}

Binomial dose-response modeling was originally formulated through a linearized weighted least squares estimation (Bliss, 1934). The linearizing transformation in this case is based on a convenient CDF form. The resulting linear model is given by:

$$
\Phi_{i}^{-1}\left(p_{i j}\right)=\beta_{0}+\beta_{1} * \operatorname{dose}_{i}+\varepsilon_{i j}
$$

Here, $\Phi^{-1}\left(p_{i j}\right)$ is the value of the inverse CDF computed for each proportion of success, $p_{i j}=$ $\mathrm{y}_{\mathrm{ij}} / \mathrm{N}$, where $\mathrm{y}_{\mathrm{ij}}$ is the number of successes at $\mathrm{j}^{\mathrm{th}}$ replication of the $\mathrm{i}^{\text {th }}$ dose, and $\mathrm{N}$ is the total number of trials. $\Phi$ is referred to as the tolerance distribution and is typically selected to be a normal distribution (probit), logistic distribution (logit), Students $t$ distribution (tobit) or gompertz distribution (gompit). $\beta_{0}$ and $\beta_{1}$ are regression parameters and $\varepsilon_{\mathrm{ij}}$ is a random error under the usual regression assumptions, i.e. $e_{i j} \sim \operatorname{NID}\left(0, \sigma^{2}\right)$. Least squares estimation of $(1)$ is carried out assuming weights proportional to $1 /\left(\mathrm{p}_{\mathrm{ij}}^{*}\left(1-\mathrm{p}_{\mathrm{ij}}\right)\right)$.

The linearized estimation has the advantages of simplicity and ease in computation, 
however, it can present problems when the expectation surface is not planar and exhibits nonlinearity. Furthermore, percentiles are obtained through inversion of predicted values which are assumed to be normally distributed and, hence, making all inferences approximate.

\subsection{Maximum Likelihood Probit Analysis}

The limitations of the linearized least squares solution were widely acknowledged early on, and a more appropriate maximum likelihood technique was proposed (Bliss, 1938; Fisher, 1935; Finney, 1971). In the maximum likelihood procedure, the underlying distribution of the data,

i.e. $y_{i j} \sim \operatorname{bin}\left(N, \pi_{i}\right)$, is used to form the likelihood function:

$$
\mathscr{L}\left(\pi_{i}\right) \propto \Pi_{i, j}\left(\pi_{i}\right)^{y_{i j}}\left(1-\pi_{i}\right)^{N-y_{i j}}
$$

The binomial parameter, $\pi_{\mathrm{i}}$, is assumed to be a function of dose given by:

$$
\pi_{i}=M+(1-M) \Phi\left(\beta_{0}+\beta_{1} \text { dose }_{i}\right)
$$

where $\Phi, \beta_{0}, \beta_{1}$, and dose $e_{i}$ are defined as in (1), and $M$ represents the natural or threshold level of the response under the control ( dose $=0$ condition). $M$ acts as a scaling factor in (3) increasing the function intercept while limiting the maximum value to be 1.0. During the estimation process, $\mathrm{M}$ can be assumed equal to 0 , fixed at a known value, or estimated in conjunction with the other parameters.

The estimation of percentile values can be derived using inversion, as above, or more typically, using a ratio of the parameters $\beta_{0}$ and $\beta_{1}$ and using a normal approximation (Fieller, 1944). Thus, while maximum likelihood improves the estimation process through the use of a correct probability distribution and a true nonlinear situation, the exact distribution for the estimated parameters and percentiles are unknown and corresponding inferences on these quantities remain approximate.

\section{Modern Estimation Techniques}

\subsection{Nonlinear Least Squares Analysis}

Dose-response estimation can be carried out by directly estimating the proportion of success as a function of dose:

$$
p_{i}=f\left(\text { dose }_{i}, \theta\right)+\epsilon_{i j}
$$

where $f\left(\operatorname{dose}_{i}, \theta\right)$ may be generalized to any continuous function of dose with a domain between 0.0 and 1.0 and parameter vector $\theta$. While this solution provides more flexibility in the functional form of the dose-response model than the probit models, for example, it fails to account for the 
underlying distribution of the data and assumes the error term, $\varepsilon_{\mathrm{ij}}$, to be normally distributed with zero mean and constant variance. This leads to asymptotic standard errors and approximate inference on $\theta$ and can produce invalid predicted values, e.g. predictions less than 0.0 or greater than 1.0. For these reasons, the above technique constitutes a poor choice for estimating doseresponse functions.

\subsection{Generalized Nonlinear Models Analysis}

A more appropriate method for nonlinear estimation is the generalized nonlinear model which expands upon the maximum likelihood approach given in section 1.2. Like that procedure, the data distribution is assumed $\mathrm{y}_{\mathrm{ij}} \sim \operatorname{bin}\left(\mathrm{N}, \pi_{\mathrm{i}}\right)$, and the likelihood function is specified as in (2). However, the binomial parameter, $\pi_{\mathrm{i}}$, may now be defined more generally as:

$$
\pi_{i}=f\left(\text { dose }_{i}, \theta\right)
$$

where $f\left(\operatorname{dose}_{i}, \theta\right)$ is as defined in nonlinear least squares (4). This strategy retains the desirable characteristics of maximum likelihood probit analysis, i.e. a true nonlinear estimation utilizing the correct data distribution, while yielding the advantage of specifying dose-response functions outside the class of CDF forms. The generalized nonlinear model specification has additional benefits. The likelihood, for example, could be redefined to include other types of data, both continuous and discrete. Furthermore, the model may include random components in a mixed model scenario to account for effects such as over dispersion due to uncertainty in dosage. If $\mathrm{f}\left(\operatorname{dose}_{\mathrm{i}}, \theta\right)$ is specified as in (3), however, the results should be identical to those of maximum likelihood probit analysis.

While generalized nonlinear models were not practical due to the lack of available computer software in the past, several computational options now exist to handle the requirements of generalized nonlinear models [see for example PROC NLMIXED in the SAS software package (SAS, 1999), or the GNLM module for the R statistical package (Lindsey, et al. 2000]. Typically, these software solutions can estimate parameter values, as well as specified functions of the parameters. Measures of variability and inference on these quantities are still approximate and are often based on the Delta method or Taylor series expansions under the usual assumptions of normality.

One useful advantage of the maximum likelihood procedures is the ability to formulate a full dummy variable specification which encompasses $\mathrm{k}$ independent treatments. This is accomplished by expanding the likelihood given in (2):

$$
\pi_{i k}=f\left(\operatorname{dose}_{i k}, \theta_{k}\right)
$$

where $\pi_{\mathrm{ik}}$ is now the binomial parameter for the $\mathrm{i}^{\text {th }}$ dose in the $\mathrm{k}^{\text {th }}$ treatment. In the generalized nonlinear model, $\pi_{\mathrm{ik}}$ can be given as:

$$
\mathscr{L}\left(\pi_{i k}\right) \propto \Pi_{i, j, k}\left(\pi_{i k}\right)^{y_{i j k}}\left(1-\pi_{i k}\right)^{N-y_{i j k}}
$$


where $\theta_{\mathrm{k}}$ is the parameter vector for the $\mathrm{k}^{\text {th }}$ treatment. Comparison of treatments in terms of the $\theta_{\mathrm{k}}$ can then be conducted through likelihood ratio tests and reduced model methodology.

\subsection{Bayesian Estimation}

The Bayesian analysis is based on the premise that given the dose-response function $\mathrm{f}\left(\operatorname{dose}_{\mathrm{i}}, \theta\right)$, the percentile of interest is simply a function of the parameter vector $\theta$, say $g(\theta)$. Hence, the estimation begins with:

$$
p\left(\theta \mid y_{i j}\right)=\frac{p\left(y_{i j} \mid \theta\right) * p(\theta)}{\int p\left(y_{i j} \mid \theta\right) * p(\theta) d \theta}
$$

where $\mathrm{p}\left(\mathrm{y}_{\mathrm{ij}} \mid \theta\right)$ is the likelihood of the data set $\mathrm{y}_{\mathrm{ij}}$ evaluated over the parameter vector $\theta, \mathrm{p}(\theta)$ is a prior distribution for $\theta$, and $\mathrm{p}\left(\theta \mid \mathrm{y}_{\mathrm{ij}}\right)$ is the posterior distribution of $\theta$ given the data $\mathrm{y}_{\mathrm{ij}}$. For the binomial case, the likelihood is equivalent to that given in (2) and the prior distribution is specified by the user based on previous experience or knowledge. If the percentile is a one-to-one function of the parameter vector $\theta$, then the posterior distribution of $g(\theta)$ may be determined from the posterior distribution of $\theta$.

Similar to the maximum likelihood procedures, the Bayesian method is a true nonlinear estimation which correctly specifies the distribution of the data. Parameter variability and inference, i.e. credible regions, are now based on exact distributions with the prior assumptions of the model. Furthermore, the Bayesian technique inherently allows for updating of the estimation procedure through augmentation of data in the likelihood.

Under the Bayesian method, higher order treatment structures may also be handled through pair-wise comparisons. This is carried out by making probability statements on the differences among treatment parameter vectors. Let $\theta_{1}$ and $\theta_{2}$ be two independent parameter values with respective posterior distributions $m\left(\theta_{1}\right)$ and $h\left(\theta_{2}\right)$. Define $Z=\theta_{1}-\theta_{2}$ as the difference in the parameter values. Then the posterior distribution for the difference in parameters, $Z$, is the transformation of variables given by:

$$
p(Z)=\int m\left(\theta_{1}\right) * h\left(\theta_{1}-Z\right) d \theta_{1}
$$

where the joint probability distribution of $\theta_{1}$ and $\theta_{2}$ is $m\left(\theta_{1}\right)^{*} h\left(\theta_{2}\right)$.

All computations and graphics were carried out using SAS (1999) or custom C program codes. Sample programs and data are available at http://www. uidaho.edu/ag/statprog. 


\section{DEMONSTRATION}

\section{Source and Description of Data}

The data used for this demonstration measure the effects of an organic pesticide on the egg hatch of black vine weevil (BVW). For each dose level, $20 \mathrm{BVW}$ eggs were placed in a petri dish containing the pesticide. Several dose levels (concentrations) were used ranging from 0.0 to $0.03 \mathrm{~g}$ of the compound. In order to obtain a complete range of doses, three independent experiments were conducted. Pooling data in this manner can conceivably result in overdispersion. This could be modeled using a mixed model methodology, however for this demonstration, the effect is considered negligible and not considered. Doses were replicated an average of 10 times and the number of unhatched eggs within a 48 hour period (success) recorded. A scatter plot of these data is shown in Figure 1. The data displayed the typical sigmoidal shape conducive to dose-response modeling.

\section{Dose-Response Model}

An appropriate model for the data in Figure 1 might be the logistic. First proposed for dose-response modeling by Berkson (1944), this model is symmetric and unimodal. It is similar to the normal distribution with the exception of heavier tails. One advantage of the logistic form is that it could be parameterized to directly estimate the $\mathrm{LC}_{50}$ percentile:

$$
\Phi=\frac{1}{\left(1+\exp \left(\beta *\left(\text { dose }_{i}-\gamma\right)\right)\right)}
$$

where $\beta$ is a rate related parameter and $\gamma$ is the $\mathrm{LC}_{50}$. The rate parameter $\beta$ can be set as positive or negative which result in decreasing or increasing sigmoidal curves, respectively. The model in (10) can be generalized to estimate the $\mathrm{Q}^{\text {th }}$ percentile as:

$$
\Phi=\frac{C}{\left(C+\exp \left(\beta *\left(\text { dose }_{i}-\gamma\right)\right)\right)}
$$

where $\mathrm{C}=(\mathrm{Q}-\mathrm{M}) /(1-\mathrm{Q}+\mathrm{M})$ and $\mathrm{M}$ is the natural threshold.

\subsection{Linearized Logit Analysis}

In order to implement (1), a logit transformation was performed on the proportions of unhatched BVW eggs, $p_{\mathrm{ij}}$, yielding the model:

$$
\operatorname{logit}\left(p_{i j}\right)=\ln \left(\frac{p_{i j}}{\left(1-p_{i j}\right)}\right)=\beta_{0}+\beta_{1} * \operatorname{dose}_{i}+\epsilon_{i j}
$$


The estimation of (12) is weighted by $1 /\left(\mathrm{p}_{\mathrm{ij}} *\left(1-\mathrm{p}_{\mathrm{ij}}\right)\right)$. The logit transformation for the conditions of $\mathrm{p}_{\mathrm{ij}}=0$ and $\mathrm{p}_{\mathrm{ij}}=1$ are undefined. This was corrected by offsetting these extremes by an arbitrarily small amount, $1 \times 10^{-7}$. While this correction should have minimal impact on the final estimation, the arbitrary nature of the offset is an additional drawback to this technique. Estimation was carried out using the simple linear regression, i.e. SAS, PROC REG (1999).

The estimated curve is shown in Figure 2 and the associated parameter estimates given in Table 1. While the parameter estimates are significant and the curve does generally follow the data, the predicted values appear shifted away from the observed data, particularly at dose $=$ $0.01 \mathrm{~g}$. The predicted $\mathrm{LC}_{50}$ value for this model (computed through inversion) is $0.00787 \mathrm{~g}$ with lower and upper $95 \%$ confidence bounds of $0.0044 \mathrm{~g}$ and $0.0108 \mathrm{~g}$, respectively.

\subsection{Maximum Likelihood Logit Analysis}

The maximum likelihood estimation maximizes (2) with the binomial parameter, $\pi_{\mathrm{ij}}$, defined as:

$$
\pi_{i}=M+(1-M) * \frac{1}{\left(1+\exp \left(\beta_{0}+\beta_{1} * \text { dose }_{i}\right)\right.}
$$

The threshold unhatched rate, $\mathrm{M}$, was considered fixed at 0.05 , for this example. This is the approximate rate expected at a dose $=0 \mathrm{~g}$. Estimation was carried out through the PROC PROBIT procedure in SAS (1999).

The fitted curve and parameter estimates are shown in Figure 3 and Table 2, respectively. Both parameters are significant and the predicted curve follows the data well. The estimated $\mathrm{LC}_{50}$ value (computed via Fieller's ratio theorem) is $0.009055 \mathrm{~g}$ with a lower $95 \%$ bound of $0.008817 \mathrm{~g}$ and an upper bound of $0.009291 \mathrm{~g}$.

\subsection{Generalized Nonlinear Logistic Analysis}

As in section 2.3, the likelihood given in (2) was maximized, however, the binomial parameter in this case was set to:

$$
\pi_{i}=M+(1-M) * \frac{1}{\left(1+\exp \left(-\beta *\left(\text { dose }_{i}-\gamma\right)\right)\right)}
$$

where $M$ was again assumed fixed at 0.05 . No random effects were specified for this model. Estimation was carried out using the PROC NLMIXED procedure in SAS (1999).

The results of the generalized nonlinear procedure are given in Table 3 . The fitted curve is shown in Figure 4. Both parameter estimates are significant and the curve follows the data well. As might be expected, these results are nearly equivalent to those for the maximum likelihood analysis, however, the model specified in (14) provides a direct estimate for the $\mathrm{LC}_{50}$ value, $\gamma$, without the use of Fieller's ratio theorem. 


\subsection{Bayesian Logistic Analysis}

Before the Bayesian estimation procedure is carried out, the logistic model given in (14) should be reparameterized such that the required prior distributions are easier to specify. Specifically, the proportion of unhatched eggs at dose $=0$, say $\theta_{1}$, reduces the model to:

$$
\theta_{1}=M+(1-M) * \frac{1}{(1+\exp (\beta * \gamma)))}
$$

and at the maximum dose, dose $_{\max }$, the proportion, $\theta_{2}$, becomes:

$$
\theta_{2}=M+(1-M) * \frac{1}{\left(1+\exp \left(-\beta *\left(\text { dose } e_{\max }-\gamma\right)\right)\right)}
$$

The domain for the parameters $\theta_{1}$ and $\theta_{2}$ lies between 0.0 and 1.0. With further consideration, it can be shown that for the $50^{\text {th }}$ percentile, $\theta_{1}$ is bounded between 0.0 and 0.5 and that $\theta_{2}$ is bounded between 0.5 and 1.0. If no prior preference is specified within these ranges, uniform priors may be specified as: $\theta_{1} \sim U(0.0,0.5)$ and $\theta_{2} \sim U(0.5,1.0)$ which are consistent with the principle of maximum entropy assuming no prior information.

Solving (15) and (16) for $\beta$ and $\gamma$, respectively, yields:

$$
\gamma^{*}=\frac{\text { dose }_{\max } * \ln \left(\left(1-\theta_{1}\right) /\left(\theta_{1}-M\right)\right)}{\ln \left(\left(1-\theta_{1}\right) /\left(\theta_{1}-M\right)\right)-\ln \left(\left(1-\theta_{2}\right) /\left(\theta_{2}-M\right)\right)}
$$

and

$$
\beta^{*}=\frac{\ln \left(\left(1-\theta_{1}\right) /\left(\theta_{1}-M\right)\right)}{\gamma^{*}}
$$

The logistic model in eq(14) can then be specified as:

$$
\pi_{i}=M+(1-M) * \frac{1}{\left(1+\exp \left(-\beta^{*}\left(\text { dose }_{i}-\gamma^{*}\right)\right)\right)}
$$

Assuming $\theta_{1}$ and $\theta_{2}$ are independent, the posterior distribution of $\theta_{1}$ and $\theta_{2}$, given the data $\mathrm{y}_{\mathrm{ij}}$, is:

$$
p\left(\theta_{1}, \theta_{2} \mid y_{i j}\right)=\frac{p\left(y_{i j} \mid \theta_{1}, \theta_{2}\right) * p\left(\theta_{1}\right) p\left(\theta_{2}\right)}{\int_{\theta_{1}} \int_{\theta_{2}} p\left(y_{i j} \mid \theta_{1}, \theta_{2}\right) * p\left(\theta_{1}\right) p\left(\theta_{2}\right) d_{\theta_{1}} d_{\theta_{2}}}
$$


The marginal distributions of $\theta_{1}$ and $\theta_{2}$ can be found through integration of the posterior using numerical or simulation (MCMC) techniques. The posterior distributions of $\beta$ and $\gamma$ can be obtained through a transformation of variables. Estimation was performed using custom $\mathrm{C}$ codes.

The estimated curve is shown in Figure 5 and the parameter estimates in Table 4 . The predicted curve is similar to that of the maximum likelihood techniques. The initial proportion of unhatched eggs, $\theta_{1}$, is estimated at 0.052179 and the final proportion at 0.999999 . Transforming these values to the $\mathrm{LC}_{50}$ value, $\gamma^{*}$, yield an estimate of $0.00906 \mathrm{~g}$. This too, is essentially equivalent to the maximum likelihood estimation. The $95 \%$ bounds are similar on the lower end, 0.00882 for Bayesian vs 0.008817 for maximum likelihood, however the Bayesian upper bound is slightly higher at 0.00930 . The estimates and bounds for $\theta_{1}, \theta_{2}$, and $\gamma^{*}$ are illustrated in the marginal posterior distributions shown in Figures 6-8. The initial and final proportions show some skewness which should be expected. The distribution for $\gamma^{*}$ (Figure 8) appears symmetric and similar to a normal distribution. This would suggest that inferences based on normal approximations used in the previous estimation techniques are reasonable.

With the exception of the linearized solution, all the estimation techniques gave similar results for both the parameter point estimates and confidence bounds. This similarity may diminish, however, if the desired percentiles change. Specifically, the more extreme percentiles may have distributions which deviate from the symmetric form. Table 5 presents the estimates and bounds for the $\mathrm{LC}_{99}$. As was seen earlier, the maximum likelihood techniques produce similar results. This is not unexpected as both software procedures use equivalent likelihoods and base their inferences on normal approximations. The Bayesian procedure, however, produces a point estimate and lower and upper credible bounds which are larger than their maximum likelihood counterparts. Given that the uniform priors used in the Bayesian estimation essentially reduce the posterior distribution to that of maximum likelihood, this difference can only arise from the underlying inferential methods. Examination of the changes in the likelihood surface reveal nonlinearity which could develop at more extreme percentiles. In these situations, the normal approximation used by maximum likelihood techniques may become less reliable. The severity of this deviation, however, will be dependent on the specific problem under investigation.

\subsection{Treatment Comparisons}

Treatment comparisons are demonstrated using the generalized nonlinear model approach. In order to simulate a second data set, the BVW data dosages were offset by adding $0.005 \mathrm{~g}$ to the original dosages. A full dummy variable model was then set up based on (14) where the parameter list was expanded to include $\beta_{1}$ and $\gamma_{1}$ for the original data, and $\beta_{2}, \gamma_{2}$ for the simulated treatment. The estimated parameter values and predicted curves are shown in Table 6 and Figure 9 , respectively. The estimated $\mathrm{LC}_{50}$ value for the original $\mathrm{BVW}$ data was $0.009 \mathrm{~g}$. As expected, the simulated treatment $\mathrm{LC}_{50}$ estimate was approximately 0.005 units larger at $0.014 \mathrm{~g}$. The rate parameter, $\beta_{\mathrm{i}}$, was 667.6 for the BVW data and 686.8 for the simulated data. Contrasts of these parameter sets indicated a significant difference in the $\mathrm{LC}_{50}$ values $(\mathrm{p}<0.0001)$ while the rate parameters were nonsignificant $(\mathrm{P}=0.7824)$. This pattern is evident from Figure 9 , where the predicted curve for the simulated data is shifted to the right of the BVW data, but the two curves 
appear parallel.

\section{CONCLUDING REMARKS}

Dose-response models have a wide range of application in agricultural research. They are useful in identifying the relative efficacy of various treatments in plant (herbicides), insect (insecticides), animal (bioassays), and environmental sciences. While traditional statistical methods have relied upon inverted solutions and asymptotic theory for inference, modern techniques such as generalized nonlinear models or Bayesian approaches provide a more flexible framework for estimation. These estimation techniques incorporate various response distributions and functional forms, and enable inferences with higher order treatment structures. Whereas both estimation methods provide similar estimates for midrange percentiles, the Bayesian estimation may be preferred when estimating extreme percentiles.

\section{REFERENCES}

Bliss, C. I. 1934. The method of probits. Science, 79:2037 pp. 38-39.

Bliss, C. I. 1938. The determination of dosage-mortality curves from small numbers. Quart. J. Pharm., 11, pp. 192-216.

Berkson, J. 1944. Application of the logistic function to bio-assay. J. Amer. Stat. Assoc. 39, pp 357-65.

Dungan, R. S., Gan, J., and Yates, S. R. 2001. Effect of temperature, organic amendment rate and moisture content on the degredation of 1,3-dichloropropene in soil. Pest Management Science, 57: 1107-1113.

Feiller, E. C. 1944. A fundamental formula in the statistics of biological assay and some applications. Quart. J. Pharm. 17, pp. 117-23.

Finney, D. J. 1971. Probit Analysis. Cambridge University Press, London.

Fisher, R. A. 1935. Appendix to Bliss, C. I.: The case of zero survivors., Ann. Appl. Biol., 22, pp. 164-5.

Hernandez-Sevillano, E., Villarroya, M., Alonso-Prados, J. L., and Garcia-Baudin, J. M. 2001. Bioassay to detect MON-37500 and Trisulfuron residues in soils. Weed Tech. 15: 447452.

Lindsey, J. K., Byrom, W. D., Wang, J., Jarvis, P., and Jones, B. 2000. Generalized nonlinear models pharmacokinetic data. Biometrics, 56, pp. 81-88. 
SAS Inst. Inc. 1999. SAS OnlineDoc, Version 8, Cary, NC.

Turner, N. D., Greene, L. W., Byers, F. M., and Kenison, D. C. 1995. Influence of incremental Zeranol implant doses on the chemical and physical characteristics of third metacarpal bone and chemical composition of liver and soft tissue from feedlot steers. J. Anim. Sci., $73: 1-8$. 
Table 1. Estimated parameters and standard errors for the linearized logistic model.

$\begin{array}{lrrl}\text { Parameter } & \text { Estimate } & \text { Std Err } & \underline{\mathbf{p}>\mathbf{t}} \\ \beta_{0} & -5.186 & 1.360 & 0.0002 \\ \beta_{1} & 658.508 & 54.914 & 0.0001\end{array}$

Table 2. Estimated parameters and standard errors for the maximum likelihood logistic model.

\begin{tabular}{lrrl} 
Parameter & Estimate & \multicolumn{1}{l}{ Std Err } & $\underline{\mathbf{p}>\mathbf{t}}$ \\
$\beta_{0}$ & -6.045 & 0.354 & 0.0001 \\
$\beta_{1}$ & 667.597 & 37.945 & 0.0001
\end{tabular}

Table 3. Estimated parameters and standard errors for the generalized nonlinear logistic model.

$\begin{array}{lrrl}\text { Parameter } & \text { Estimate } & \text { Std Err } & \underline{\mathbf{p}>\mathbf{t}} \\ \gamma & 0.0091 & 0.0001 & 0.0001 \\ \beta & 667.600 & 37.945 & 0.0001\end{array}$

Table 4. Estimated parameters and 95\% confidence regions for the Bayesian logistic model.

\section{$\underline{\text { Credible Regions }}$}

$\begin{array}{lrrr}\text { Parameter } & \text { Estimate } & \text { Lower } & \text { Upper } \\ \theta_{1} & 0.0521 & 0.05156 & 0.05549 \\ \theta_{2} & 0.9999 & 0.99990 & 1.00000\end{array}$


Table 5. Estimated $\mathrm{LC}_{99}$ values and their corresponding $95 \%$ confidence bounds for the maximum likelihood (ML), generalized nonlinear model (GNLM), and Bayesian (Bayes) methods.

\begin{tabular}{lcccc} 
& \multicolumn{2}{c}{ LC $_{99}$} & \multicolumn{2}{c}{$\mathbf{9 5 \%}$ Bounds } \\
Method & & Estimate & Lower & Upper \\
ML & 0.01593 & 0.01521 & 001683 \\
GNLM & 0.01588 & 0.01508 & 0.01667 \\
Bayes & 0.01626 & 0.01548 & 0.01710
\end{tabular}

Table 6. Estimated logistic model parameters and standard errors for the actual (Treatment 1) and simulated (Treatment 2) black vine weevil data.

$\begin{array}{llrrrr}\text { Treatment } & \text { Parameter } & \text { Estimate } & \text { Std Error } & \underline{p}>\chi^{2} \\ 1 & \beta_{1} & 475.51 & 18.33 & 0.0001 \\ & \gamma_{1} & 0.00863 & 0.00013 & 0.0001 \\ 2 & \beta_{2} & 417.44 & 16.77 & 0.0001 \\ & \gamma_{2} & 0.01366 & 0.00015 & 0.0001\end{array}$




\section{Applied Statistics in Agriculture}

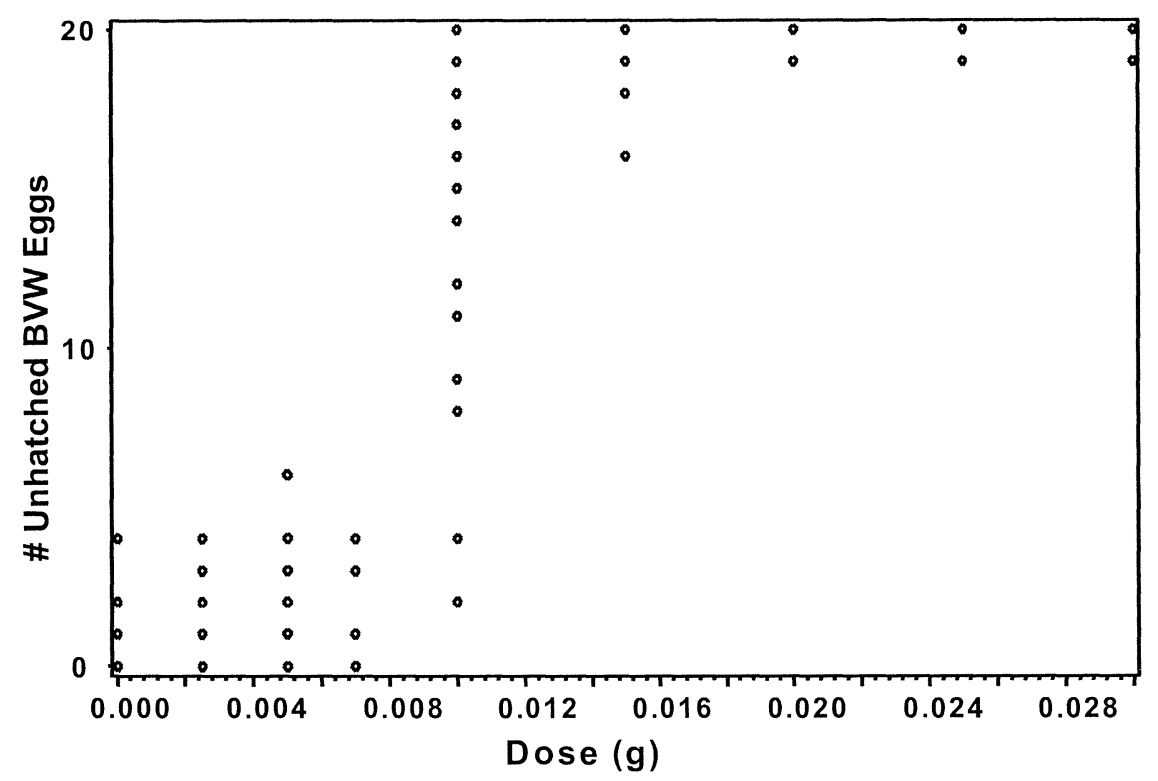

Figure 1. Scatter plot of the number of unhatched Black Vine Weevil (BVW) eggs versus dosage of an organic pesticide.

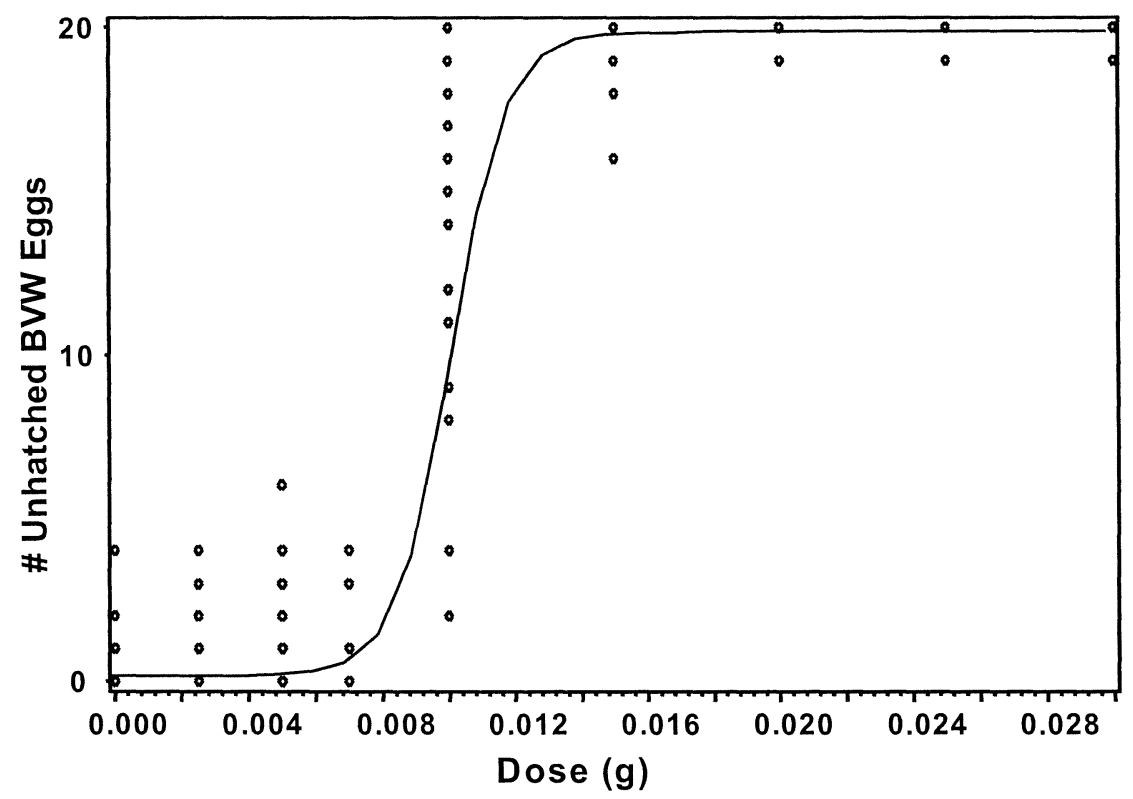

Figure 2. Estimated linearized logistic model for the BVW eggs. 


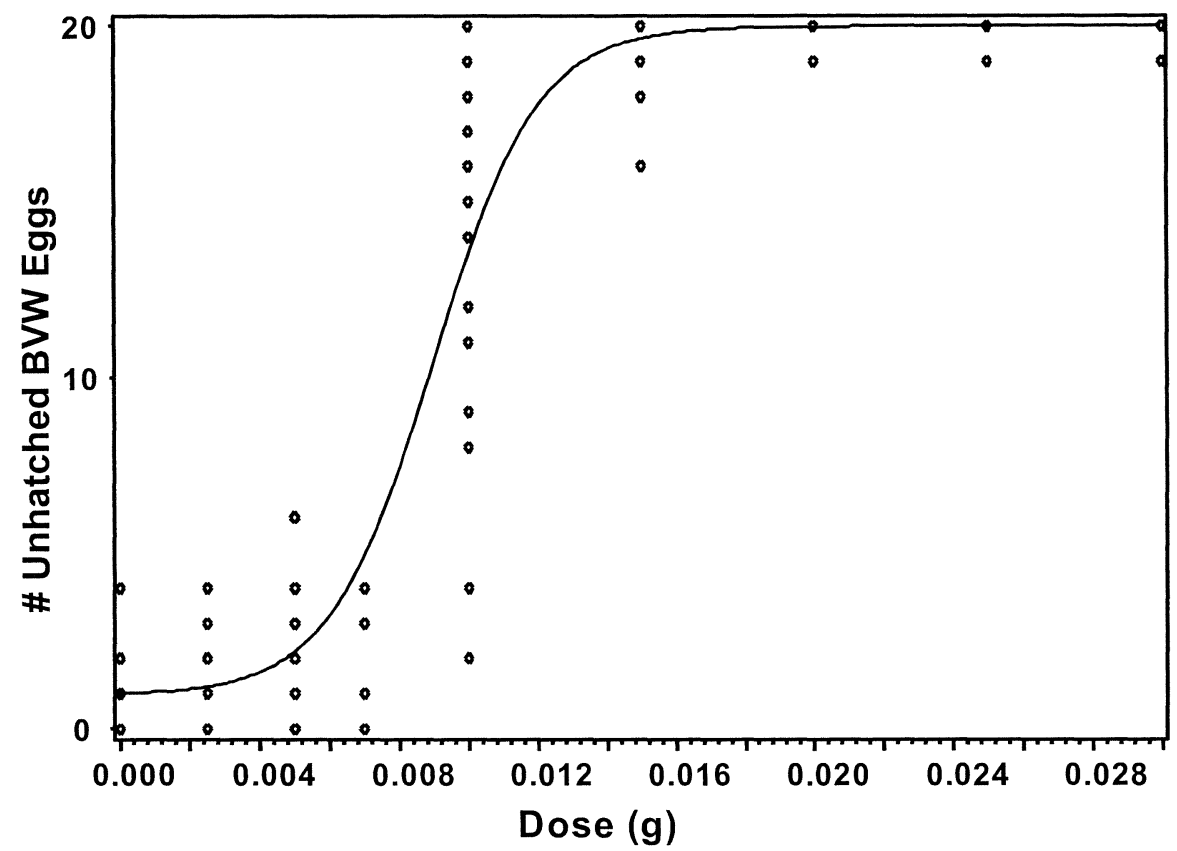

Figure 3. Estimated maximum likelihood logistic model for the BVW eggs.

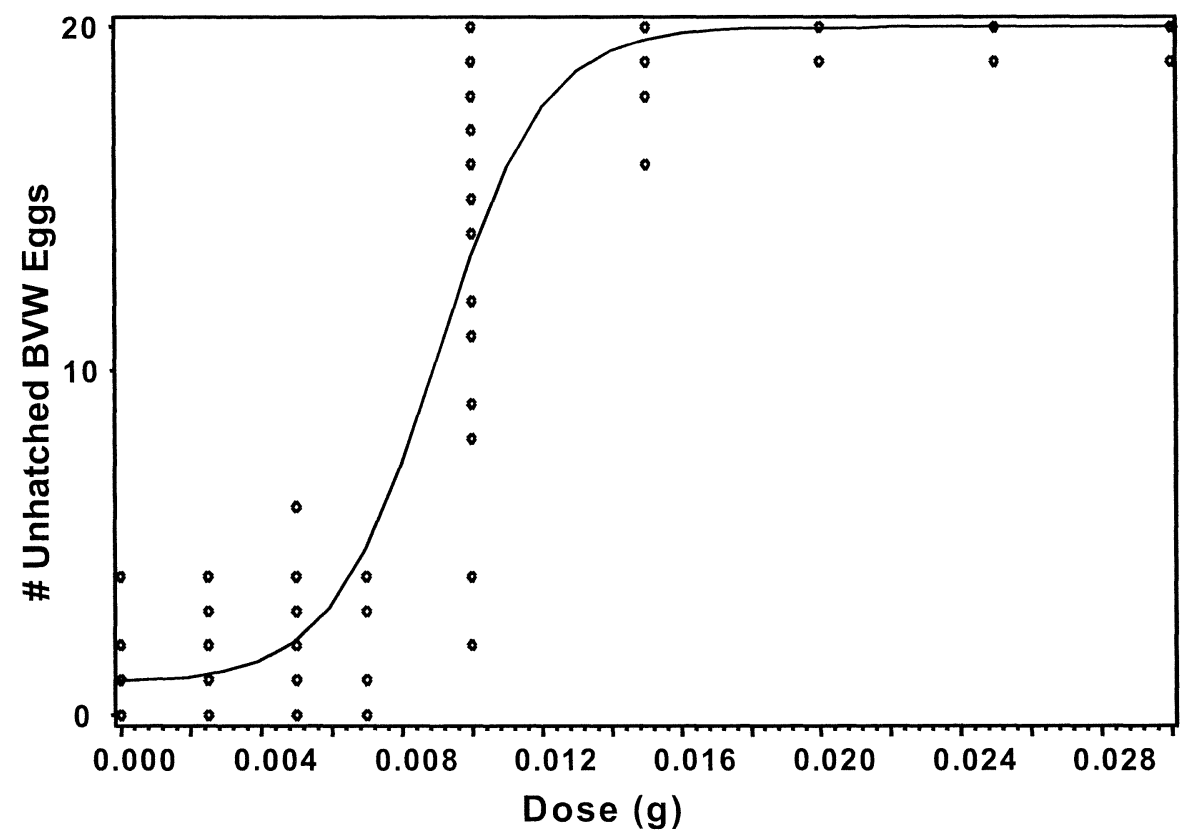

Figure 4. Estimated generalized nonlinear logistic model for the BVW eggs. 


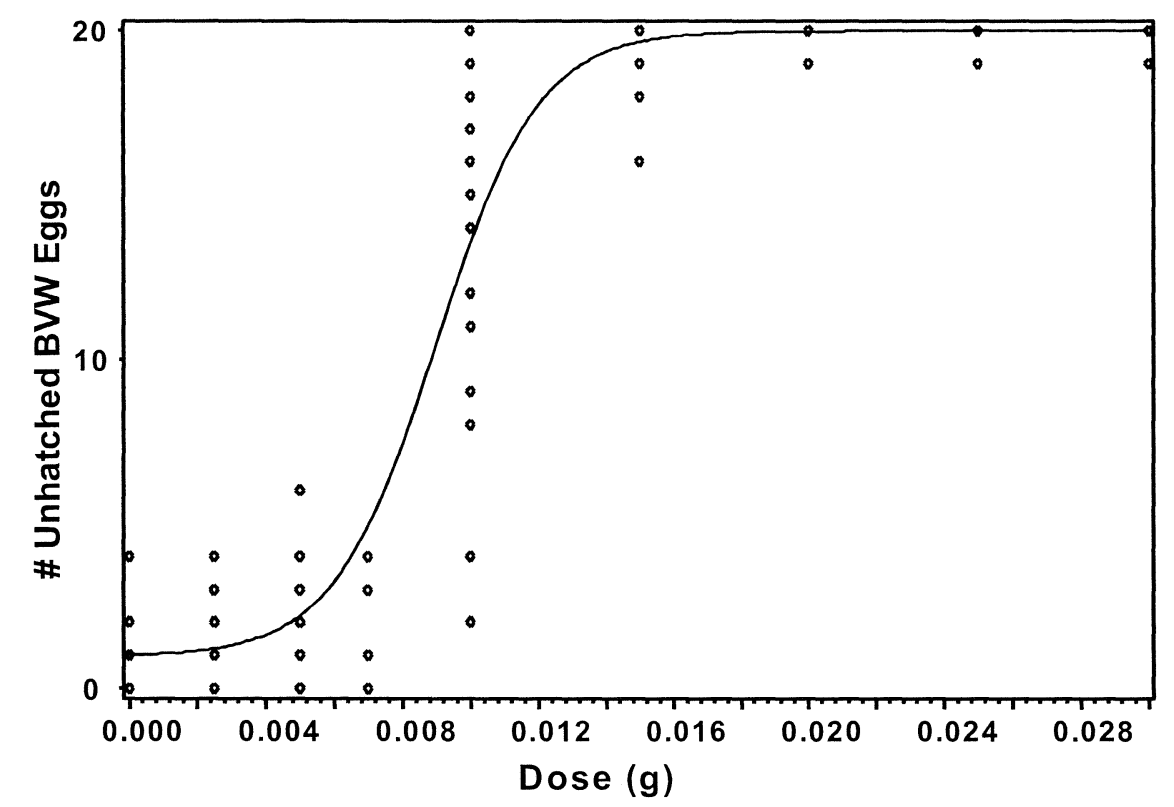

Figure 5. Estimated Bayesian logistic model for the BVW eggs.

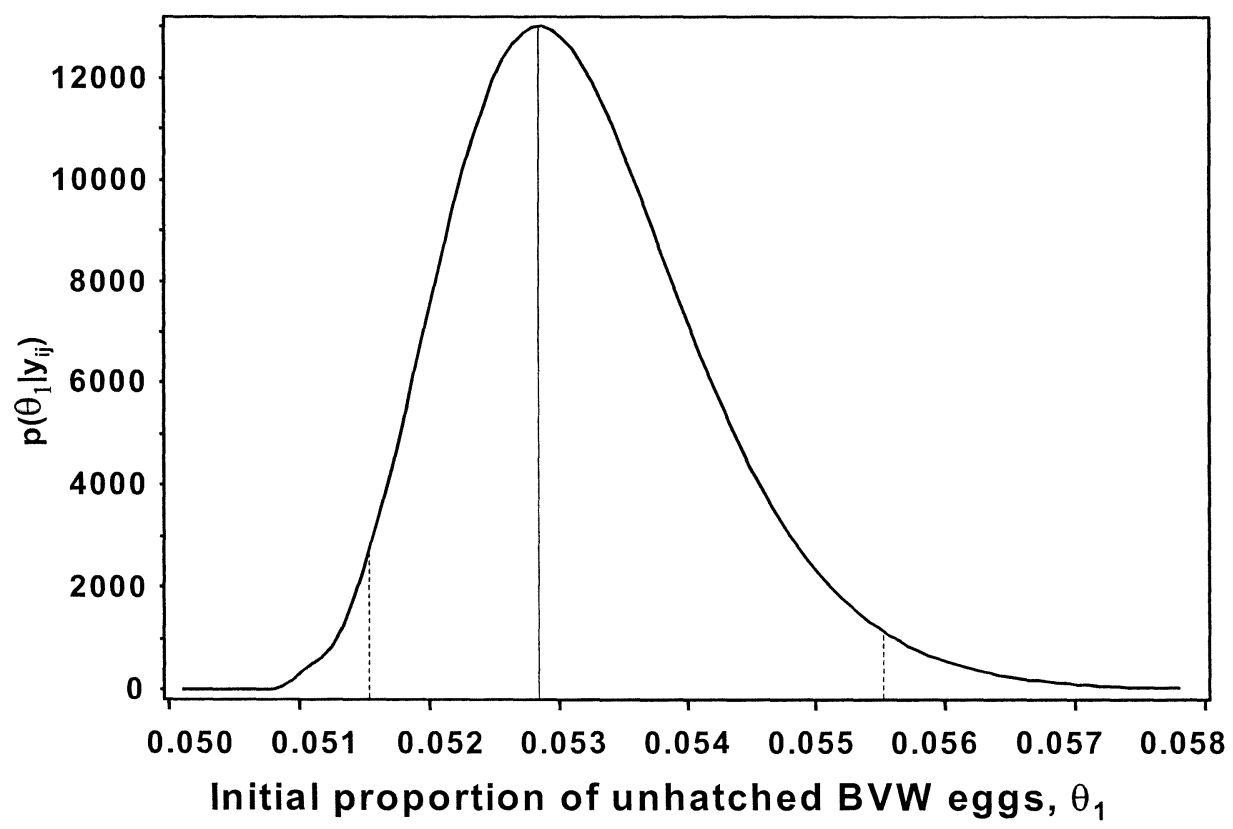

Figure 6. Posterior probability distribution for the initial proportion of unhatched BVW eggs, $\theta_{1}$. Solid vertical line indicates the most probable value, while the dashed lines indicate $95 \%$ credible bounds. 


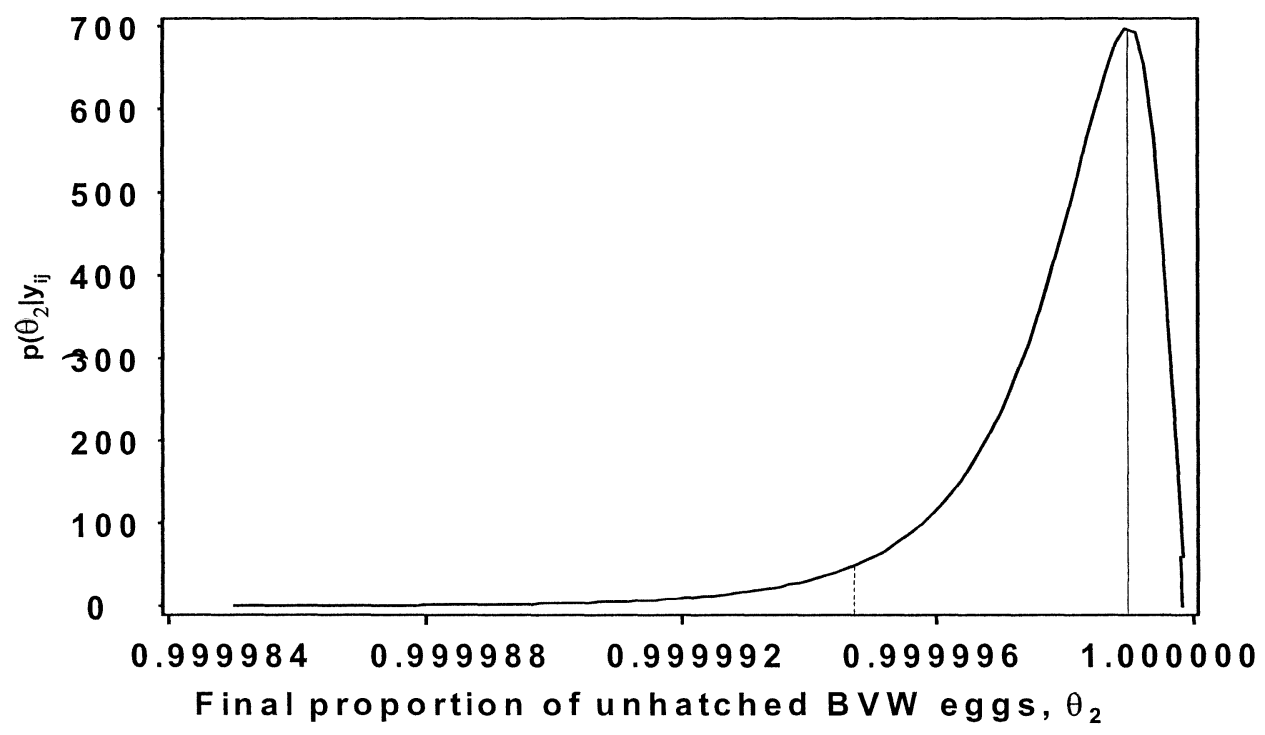

Figure 7. Posterior probability distribution for the final proportion of unhatched BVW eggs, $\theta_{2}$. Solid vertical line indicates the most probable value, while the dashed lines indicate $95 \%$ credible bounds.

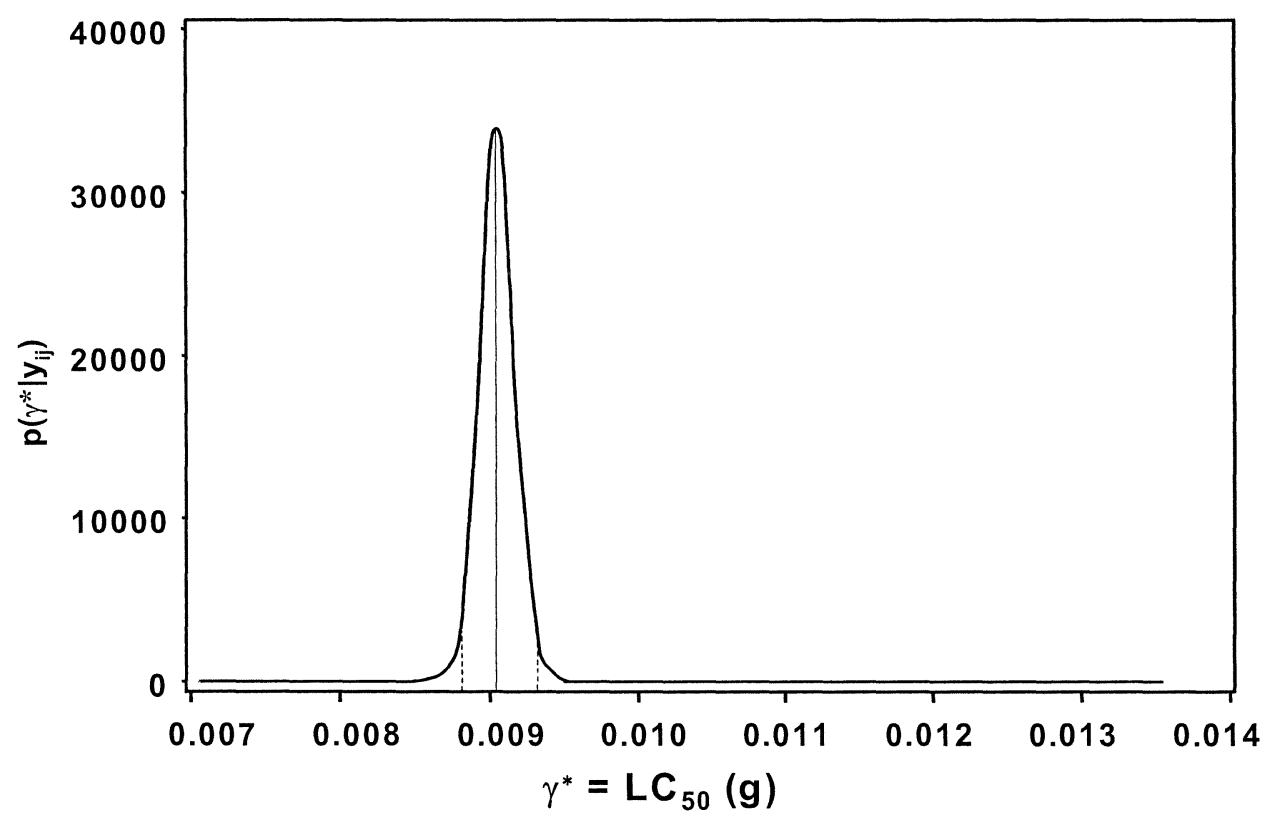

Figure 8. Posterior probability distribution for the $\mathrm{LC}_{50}$ of BVW eggs, $\gamma^{*}$. Solid vertical line indicates the most probable value, while the dashed lines indicate $95 \%$ credible bounds. 


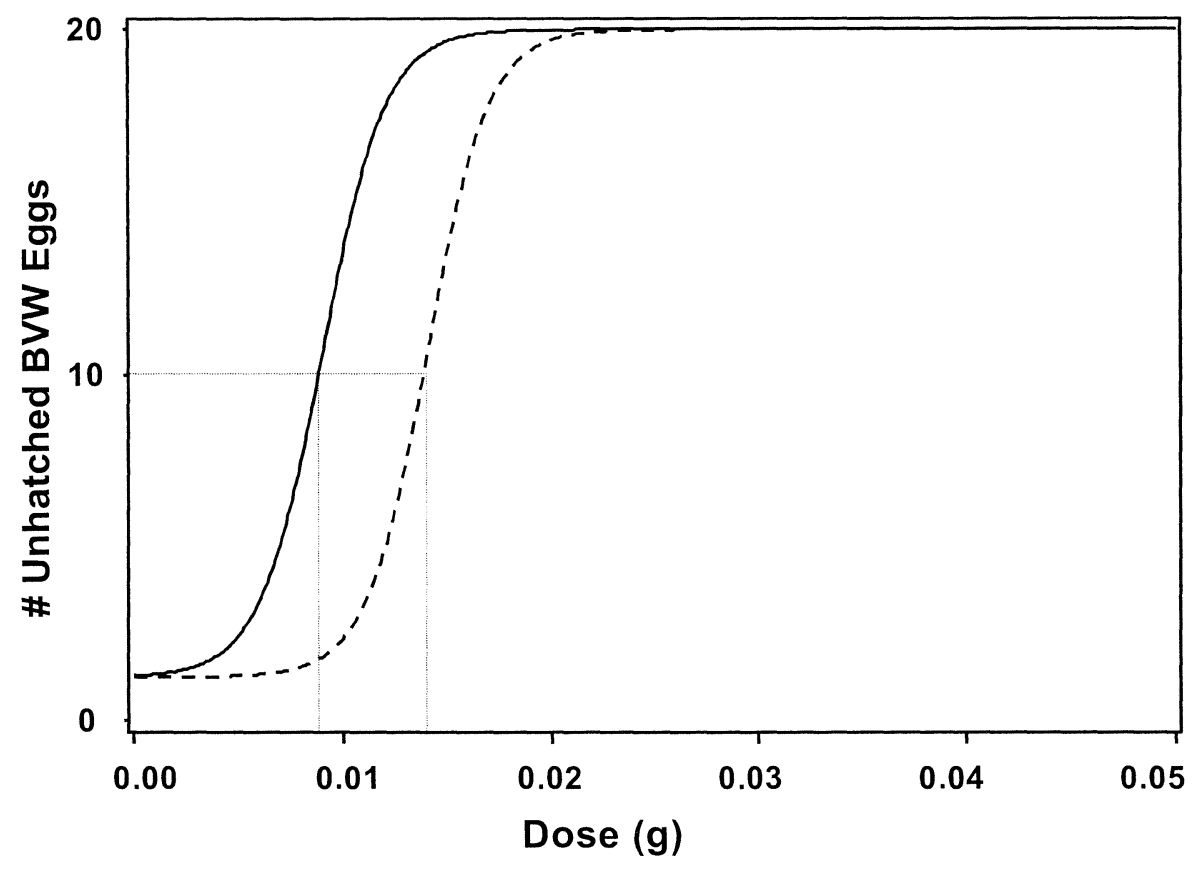

Figure 9. Estimated logistic curves for the original (solid line) and simulated (large dashed line) BVW data. Smaller dashed lines indicate the estimated $\mathrm{LC}_{50}$ of each curve. 